"This is the accepted version of the following article: Alexandra Raevskaya, Vladimir Lesnyak, Danny Haubold, Volodymyr Dzhagan, Oleksandr Stroyuk, Nikolai Gaponik, Dietrich R. T. Zahn, Alexander Eychmüller (2017). A Fine Size Selection of Brightly Luminescent Water-Soluble AgIn-S and Ag-In-S/ZnS Quantum Dots. J. Phys. Chem. C, 2017, 121 (16), pp 9032-9042, which has been published in final form at DOI: 10.1021/acs.jpcc.7b00849"

\title{
A Fine Size Selection of Brightly Luminescent Water-Soluble Ag-In-S and Ag-In-S/ZnS Quantum Dots
}

\author{
Alexandra Raevskaya ${ }^{a}$, Vladimir Lesnyak $^{b}$, Danny Haubold $^{b}$, Volodymyr Dzhagan, $^{c, d}$, \\ Oleksandr Stroyuk ${ }^{a, b^{*}}$, Nikolai Gaponik ${ }^{b^{*}}$, Dietrich R. T. Zahn ${ }^{c}$, Alexander Eychmüller ${ }^{b}$ \\ ${ }^{a}$ L.V. Pysarzhevsky Institute of Physical Chemistry, \\ National Academy of Sciences of Ukraine, Kyiv, 03028, Ukraine \\ ${ }^{\boldsymbol{b}}$ Physical Chemistry, TU Dresden, 01062 Dresden, Germany \\ ${ }^{c}$ Semiconductor Physics, Chemnitz University of Technology, 09107 Chemnitz, Germany \\ ${ }^{d}$ V. E. Lashkaryov Institute of Semiconductors Physics, \\ National Academy of Sciences of Ukraine, Kyiv, 03028, Ukraine
}

Authors for correspondence:

${ }^{*}$ Dr. Oleksandr Stroyuk, Physikalische Chemie, Technische Universität Dresden, Bergstraße 66b, 01062 Dresden, Germany, Tel. $+49(0) 351463$ 34351; Laboratory of Organic Photovoltaics and Electrochemistry, L.V. Pysarzhevsky Institute of Physical Chemistry, National Academy of Sciences of Ukraine, Kyiv, Prosp. Nauky 31, 03028, Ukraine, Tel. +38(0) 4452502 70, e-mail: alstroyuk@ukr.net; oleksandr.stroyuk@chemie.tu-dresden.de *Prof. Nikolai Gaponik, Physikalische Chemie, Technische Universität Dresden, Bergstraße 66b, 01062 Dresden, Germany, Tel. +49(0)351 463 35203, Fax: +49(0)351 463 37164, e-mail: nikolai.gaponik@chemie.tu-dresden.de 
Abstract A size-selected series of water-soluble luminescent Ag-In-S (AIS) and core/shell AIS/ZnS QDs were produced by a precipitation technique. Up to 10-11 fractions of size-selected AIS (AIS/ZnS) QDs emitting in a broad color range from deep-red to bluish-green were isolated with the photoluminescence (PL) quantum yield reaching $47 \%$ for intermediate fractions. The size of the isolated AIS (AIS/ZnS) QDs varied from $\sim 2 \mathrm{~nm}$ to $\sim 3.5 \mathrm{~nm}$ at a roughly constant chemical composition of the particles throughout the fractions as shown by the X-ray photoelectron spectroscopy.

The decrease of the mean AIS QD size in consecutive fractions was accompanied by an increase of the structural QD imperfection/disorder as deduced from a notable Urbach absorption "tail" below the fundamental absorption edge. The Urbach increased from 90-100 meV for the largest QDs up to $350 \mathrm{meV}$ for the smallest QDs, indicating a broadening of the distribution of sub-bandgap states. Both the Urbach energy and the PL bandwidth of the size-selected AIS QDs increased with QD size reduction from $3-4 \mathrm{~nm}$ to $\sim 2 \mathrm{~nm}$ and a distinct correlation was observed between these parameters.

A study of size-selected AIS and AIS/ZnS QDs by UV photoelectron spectroscopy on Au and FTO substrates revealed their valence band level $E_{\mathrm{VB}}$ at $\sim 6.6 \mathrm{eV}$ (on $\mathrm{Au}$ ) and $\sim 7 \mathrm{eV}$ (on FTO) and pinned to the Fermi level of conductive substrates resulting in a masking of any possible sizedependence of the valence band edge position. 


\section{Introduction}

Quantum dots (QDs) of ternary metal chalcogenides, in particular, $\mathrm{CuInS}_{2}$ (CIS) and $\mathrm{AgInS}_{2}$ (AIS) can be valuable alternatives to binary cadmium and lead chalcogenide QDs for applications in light-emitting and light harvesting systems, such as LEDs, bio-diagnostic tools, and solar cells ${ }^{1-5}$. CIS and AIS QDs revealed high absorption coefficients in the visible spectral range as well as quite high photoluminescence (PL) quantum yields (QY) reaching $\sim 60 \%$ after passivation with protective shells such as $\mathrm{ZnS}^{1,3-7}$. CIS and AIS QDs were successfully exploited as light harvesters in solar cells with the highest reported efficiency of $\sim 12 \%^{2-4,6,8}$.

Ternary indium-chalcogenide-based QDs offer special features that cannot be observed for binary cadmium and lead chalcogenide nanocrystals, in particular, the QD structure tolerance to composition variations, non-stoichiometry, and cationic doping $g^{3,4,6,9,10}$. The preservation of the crystalline structure in a broad range of QD compositions allows the band gap and optical properties of ternary QDs to be tuned simply by changing the ratio of copper (silver) to indium to chalcogen. The PL maximum, for instance, can be tuned between the visible and the near-IR spectral range.

Another special feature of the CIS (AIS) QDs is the large width of their PL emission. Similarly to cadmium and lead chalcogenides, the ternary QDs smaller than 5-6 nm exhibit distinct size dependences of absorption and PL characteristics as a result of spatial confinement of the charge

carriers $^{5,7,9-11}$. However, contrary to the defect state-related broadband emission observed for cadmium and lead chalcogenide QDs, the broadband PL of ternary QDs is characterized by a high QY and notable size dependence of the PL maximum ${ }^{4,7,9}$.

As the composition of the ternary QDs can be simply controlled by the synthesis conditions, most studies typically focus on their composition-dependent optical and/or photovoltaic properties. Reports on size-selected series of ternary QDs are far less numerous ${ }^{12-18}$. However, these studies showed that the size variation of ternary QDs is also an efficient tool for influencing their optoelectronic properties, especially in the critical range of sizes smaller than 3-4 nm, where strong confinement of the charge carriers is expected for most ternary metal chalcogenide QDs ${ }^{19}$.

The ternary QDs with a definite size and size distribution are typically synthesized in colloidal solutions, where the rates of nucleation and growth of the primary nuclei can be reliably controlled. Similarly to cadmium and lead chalcogenide QDs, the most precise control over the CIS (AIS) QD structure and morphology was achieved by using the well-reported heating-up and hot-injection syntheses in organic solvents with a high boiling point ${ }^{2,5-7,9,10}$. However, many potential applications of the ternary QDs, in particular as luminescing biomarkers, require the QDs to be soluble in polar solvents, particularly in water. The QD transfer into aqueous solutions is typically performed by exchanging the primary liophilic ligands with smaller bifunctional molecules, such as thioglycolic 
(mercaptoacetic) or mercaptopropionic acid that can passivate the QD surface and, at the same time, make them soluble in polar media ${ }^{2,6,9-11,20}$. Alternatively, attempts were made to produce ternary QDs

directly in water and other polar solvents ${ }^{21-25}$. Such approaches appeared to be more or less successful for the production of CIS/AIS QD inks for solar cells $\mathrm{s}^{6,9,10,11,18,22,25}$ and photocatalysis ${ }^{21}$. However, the PL characteristics of the ternary QDs produced by the direct aqueous synthesis approaches still remain largely inferior to those of the CIS (AIS) QDs synthesized in organic oils and then transferred into water by ligand exchange $e^{6,9,10}$.

The size-selected series of various semiconductor QDs can be produced by their selective precipitation from original polydisperse ("crude") colloids. The precipitation is induced by a measured addition of a "poor" solvent that mixes freely with the colloidal solution but decreases the aggregation stability of the QDs. The aggregation involves the largest QDs in the present ensemble that flocculate and precipitate, while smaller QDs remain dispersed in the colloidal solution. The QDs precipitate and can be collected and re-dispersed and the entire procedure repeated many times to produce a series of size-selected QDs. Such a method became "classical” for the size-selective separation of cadmium chalcogenide $\mathrm{QDs}^{26-39}$, but it also revealed general applicability and was successfully used for other semiconductors, such as $\mathrm{Si}^{40}, \operatorname{InP}^{31,41}, \operatorname{InAs}^{38}, \mathrm{CuInS}_{2}{ }^{7,41-44}, \mathrm{CuGaS}_{2}{ }^{45}, \mathrm{AgIn}(\mathrm{Ga}) \mathrm{S}_{2}{ }^{46}$, and $\mathrm{Cu}_{2} \mathrm{ZnSnS}_{4}$ nanoparticle colloids ${ }^{47,48}$. The reported examples of the size-selective precipitation of ternary QDs are largely limited to colloids produced by the hot injection/heating up methods.

Recently, we reported the synthesis of AIS QDs stabilized in water by mercaptoacetic acid $(\mathrm{MAA})^{24}$ and polyethyleneimine ${ }^{49}$. The strongly emitting QDs were prepared by a facile and reproducible method with the potential of scaling-up. In this paper, we show the feasibility of sizeselective fractionation of MAA-stabilized Ag-In-S QD colloids for the preparation of QD series with relastively bright and multi-color emission. This report focuses on the optical properties of the sizeselected AIS QDs as well as on the relationships between their absorption and emission characteristics.

\section{Material and methods}

Aqueous colloidal solutions of AIS QDs were prepared by a reaction between sodium sulfide and a mixture of silver(I) and indium(III) mercaptoacetate (MA) complexes according to a reported procedure $^{24}$. In a typical synthesis, the starting (feed) molar ratio of silver to indium to sulfur, (Ag:In:S $)_{0}$, was adjusted to 1:7:10. A detailed description of the synthetic procedure can be found in Supporting Information (SI). For comparison purposes, binary silver sulfide and indium sulfide colloids were prepared by the same procedure. 
The core/shell AIS/ZnS QDs were produced from AIS QDs by adding zinc(II) and MAA, typically at a molar zinc to silver ratio of $(\mathrm{Zn}: \mathrm{Ag})_{0}=10: 1$. Then, the solutions were additionally heated to $\sim 98^{\circ} \mathrm{C}$ for $30 \mathrm{~min}$ to decompose a $\mathrm{Zn}-\mathrm{MAA}$ complex and to form a $\mathrm{ZnS}$ shell.

The as-prepared AIS or AIS/ZnS QD colloids were concentrated by a factor of $\sim 15$ on a rotary evaporator at around $40^{\circ} \mathrm{C}$. The concentrated AIS QD colloids are further denoted as "crude colloids".

In a typical size-selection procedure, to $10 \mathrm{~mL}$ of crude colloids $2.5 \mathrm{~mL}$ of 2-propanol were added to initiate aggregation of the QDs with subsequent centrifugation at $4500 \mathrm{rpm}$ for $5 \mathrm{~min}$. The precipitate was separated and designated further as fraction \#1. Then, a fresh smaller portion of 2propanol $(0.5 \mathrm{~mL})$ was added resulting again in the formation of turbid solution which also was centrifuged at $4500 \mathrm{rpm}$ for $5 \mathrm{~min}$ and the precipitate was collected. This procedure was repeated 6 times with $0.5 \mathrm{~mL}$ of 2-propanol to produce precipitates that were designated as fractions \#2-7. The following two fractions (\#8 and \#9) of the QD precipitate were obtained by a similar procedure using $1.0 \mathrm{~mL}$ 2-propanol and finally the two last fractions, \#10 and \#11 were selected by adding $2 \mathrm{~mL}$ of 2propanol. Precipitates of MAA-capped silver and indium sulfide QDs were also produced for comparison in a similar way by adding $10.0 \mathrm{~mL}$ of 2-propanol to $10.0 \mathrm{~mL}$ of crude solutions and dissolved in $10.0 \mathrm{~mL}$ of Milli-Q water. The collected precipitates \#1-11 were dissolved in $1 \mathrm{~mL}$ of Milli$\mathrm{Q}$ water and stored in the dark at room temperature. For absorption and PL measurements the AIS and AIS/ZnS QD solutions were diluted by a factor of 100-500 (depending on the fraction number) by Milli-Q water. The above-described typical size-selection procedure can be applied to the crude AIS QDs of any starting composition and yields QD series with highly reproducible optical characteristics. Also, to produce a larger amount of the size-selected QDs we scaled the precipitation procedure up to $200 \mathrm{~mL}$ of starting concentrated crude solution without any observable variation in the optical properties of the sizeselected QD series.

The details of absorption, PL, X-ray photoelectron (XPS) and UV photoelectron (UPS) spectra acquisition, PL QY determination, as well as the experiments of X-ray diffraction (XRD) and transmission electron microscopy (TEM) can be found in SI.

\section{Results and Discussion}

Preparation of crude AIS and AIS/ZnS QD colloids for the size-selective precipitation. In the present paper we adopted a synthesis of aqueous MAA-stabilized AIS QDs similar to ref. 24 using MAA complexes of $\mathrm{Ag}(\mathrm{I}), \operatorname{In}(\mathrm{III})$, and $\mathrm{Zn}(\mathrm{II})$ as precursors and MAA ions as a stabilizer for the AIS and AIS/ZnS QDs. The composition of AIS and AIS/ZnS QDs depends on the feed ratios of the components, (Ag:In:S $)_{0}$ and $(\mathrm{Zn}: \mathrm{Ag})_{0}$. As seen from Fig. S2, curve 1 (SI), no PL can be detected for 
colloidal solutions produced either without silver or indium. The introduction of $\operatorname{Ag}(\mathrm{I})$ results in the formation of AIS QDs emitting in a broad range with PL maxima at 580-770 nm depending on the silver to indium ratio (SI, Fig. S2, curves 2-8). A maximal PL intensity, as well as the highest stability, were shown by the QD solutions prepared at an Ag:In ratio in the range of 1:5 to 1:8. A variation of the In content at a constant silver to sulfide ratio also revealed that the maximal PL intensity can be achieved at $(\mathrm{Ag}: \mathrm{In}: \mathrm{S})_{0}$ in the range of 1:6:10 to 1:8:10 (SI, Fig. S3). The observations of the aggregation stability of the AIS colloids indicated that the most stable systems were formed at $(\mathrm{Ag}: \operatorname{In}: \mathrm{S})_{0}=1: 7: 10$ and this ratio was used in further syntheses.

The PL intensity of crude AIS-MAA colloids was found to increase strongly upon postsynthesis heating of the colloidal solutions at $96-98{ }^{\circ} \mathrm{C}$ with the PL band position and width not being affected (SI, Fig. S4). The highest PL enhancement was observed for the first $30 \mathrm{~min}$ heating and therefore this procedure as a routine for the preparation of crude AIS QD colloids.

To increase the stability of the AIS QDs against oxidation/aggregation, they were covered with a protective $\mathrm{ZnS}$ shell by thermal decomposition of zinc(II) mercaptoacetate on the QD surface similarly to the previously studied $\mathrm{AIS} / \mathrm{ZnS}^{24}$ and $\mathrm{CIS} / \mathrm{ZnS}^{25}$ QDs. The PL intensity increases upon deposition of a $\mathrm{ZnS}$ shell more than twice already at a zinc to silver ratio of $(\mathrm{Zn}: \mathrm{Ag})_{0}=1: 1$ and continues to grow with higher $\mathrm{ZnS}$ contents up to $(\mathrm{Zn}: \mathrm{Ag})_{0}=10: 1$ (SI, Fig. S5). Simultaneously the PL band maximum continuously shifts from $\sim 650 \mathrm{~nm}$ to $\sim 610 \mathrm{~nm}$ as a result of the well-reported effect of unintentional doping/alloying of the core material with $\mathrm{Zn}$ (II) ions ${ }^{1,3,6,9}$. The effect of alloying prevails over the effect of weakening of the quantum confinement in the core/shell structures, which should lead to a red shift of both PL and absorption spectra ${ }^{1,3,4}$. At a Zn-MAA content higher than $(\mathrm{Zn}: \mathrm{Ag})_{0}=$ 10:1 only a small blue shift and no further PL increase were observed (Fig. S5) indicating the saturation of both the $\mathrm{Zn}^{2+}$ doping and the degree of passivation of AIS QDs. We chose $(\mathrm{Zn}: \mathrm{Ag})_{0}=$ 10:1 as an optimal ratio for further syntheses of core/shell AIS/ZnS QDs.

Finally, the crude AIS and AIS/ZnS QD solutions were subjected to rotary evaporation. This treatment was found to affect the PL properties of the QDs. For example, in the case of bare AIS QDs a concentration increase during the rotary evaporation resulted in a red shift of both the PL maximum (SI, Fig. S1, curves 1 and $1^{\prime}$ ) and the absorption band position indicating additional growth of the particles. At the same time, the PL band maximum of the core/shell AIS/ZnS QDs experienced a blue shift upon rotary evaporation of the solvent indicating some increase in the level of $\mathrm{Zn}$ (II) doping of the core QDs (SI, Fig. S1, curves 2 and $2^{\prime}$ ).

Size-selective fractionation of crude AIS and AIS/ZnS QD colloids. Similarly to the earlier reported aqueous MAA-stabilized cadmium chalcogenide $\mathrm{QDs}^{28,36-38}$, AIS colloids become unstable upon the addition of a "poor" solvent - 2-propanol and are prone to fast aggregation. Similarly to the cadmium chalcogenides and other colloidal semiconductor QDs, we expected that such a procedure 
will allow us to separate the QDs by their size. Presumably, in the case of the ternary AIS QDs, a dependence of the QD stability on their composition can be envisaged as well, thus distorting the "pure" size-selective precipitation. However, the results of X-ray photoelectron spectroscopy (XPS) of the size-selected AIS and AIS/ZnS QDs reported below, showed that the precipitation of the ternary QDs is governed mostly by their size, not by the composition and occurs similarly to other nanoparticles as reported earlier ${ }^{28,36-38}$.

The first portion of 2-propanol should be relatively large $(2.5 \mathrm{~mL}$ for $10 \mathrm{~mL}$ of the concentrated QD solution) to induce the precipitation of the QD fraction \#1. This fraction could be readily dispersed in water but the resulting colloid was characterized by a low PL efficiency. As revealed by TEM (not shown) precipitate \#1 contained large aggregates of nanoparticles formed, most probably, at the solution/air interface during the rotary evaporation. After elimination of fraction \#1, the QD precipitation could be continued by adding relatively small amounts of 2-propanol $(0.5 \mathrm{~mL})$. Each addition resulted in the formation of a precipitate that can be separated and re-dispersed in water. After 5-6 repetitions of this procedure the 2-propanol volume was increased to $1.0 \mathrm{~mL}$ for fractions \#8 and $\# 9$ and finally to $2.0 \mathrm{~mL}$ - for fractions \#10 and \#11. As the precipitates were dissolved in the same volumes of water, the integral absorbance of the resulting colloids reflected directly the amount of QDs of a given sort in the general QD ensemble present in the crude colloid. Figure 1a (upper panel) shows that the mass of the precipitated QDs was maximal for fraction \#3, then decreased constantly as the precipitation was continued.

In the case of core/shell AIS/ZnS QD colloids the precipitation/re-dispersion procedure yielded a series of quite strongly luminescing colloidal solutions with spectacularly different PL colors, varying from deep red for fraction \#2 through orange for \#5-7, to yellow for \#9 and, finally to green and bluish-green for the least populated fractions \#10 and \#11 (Fig. 1a, lower panel).

XRD of size-selected QDs. The XRD patterns of fractionated AIS QDs reveal several strongly broadened reflections, the three most intense of them are observed at $26.0^{\circ}, 44.7^{\circ}$, and $52.2^{\circ}$ (Fig. 1b). The positions are typical for the (112), (220), and (312) planes of tetragonal chalcopyrite (JCPDS \# 251330), however, a contribution of other AIS phases, such as, for example, In-rich cubic $A g \operatorname{In}_{5} \mathrm{~S}_{8}$ (JCPDS \# 25-1329) cannot be either ruled out or confirmed due to the large width of the reflections. The XRD patterns of the precipitated QDs are essentially the same for all fractions (curves 2-10, Fig. $1 b)$, differing only in the peak width and intensity. SImilar XRD patterns were also observed for the core/shell AIS/ZnS QDs (SI, Fig. S6a). Also, the XRD patterns of the QDs differ drastically from those of individual silver and indium sulfides prepared under the similar conditions (SI, Fig. S6b).

The large width of the XRD reflections of the precipitated QDs indicates their small size. Estimations made using the Scherrer equations showed that fraction \#2 is composed of QDs as small as $2.8 \mathrm{~nm}$ and the QD size is further reduced to $2.3 \mathrm{~nm}$ for fractions \#10 and \#11. Similar results, 3.0 
$\mathrm{nm}$ for fraction \#2 and $2.2 \mathrm{~nm}$ for \#10 were obtained for the series produced from another crude AIS QD colloid, synthesized at (Ag:In:S $)_{0}=1: 7: 5$ (XRD patterns not shown) thus confirming a general trend of the size variation with the AIS QD fraction number. As the size of QDs decreases, the intensity of the XRD reflections lowers indicating an increase of average disordering of the crystalline structure of the precipitated QDs, which is quite expectable for such small particles.

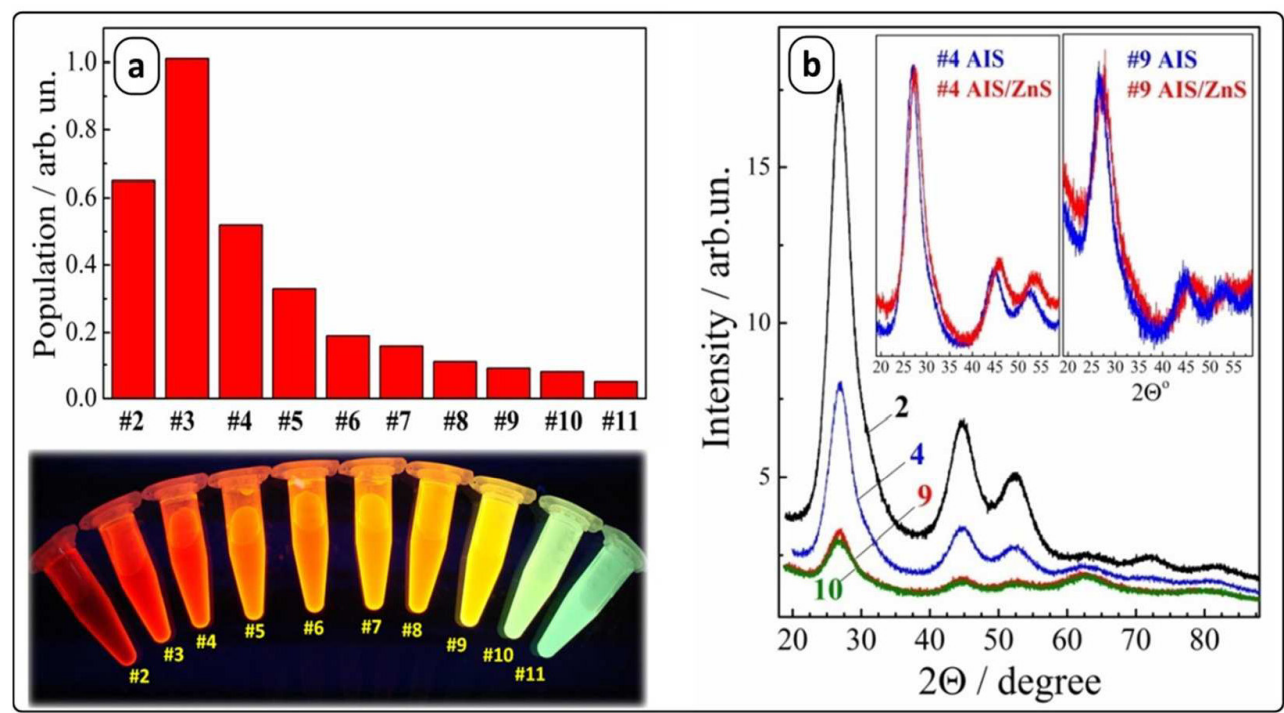

Figure 1. (a) Relative population of precipitated AIS QD fractions (upper panel) and a photograph of colloidal AIS/ZnS solutions produced by re-dispersion of the precipitated fractions (number is given under the image) in water. (b) XRD patterns of size-selected AIS QDs. Curve numbers correspond to the numbers of fractions. Insert: normalized XRD patterns for AIS and AIS/ZnS QDs from fractions \#4 (left insert panel) and \#9 (right insert panel). (Ag:In:S) $)_{0}=1: 7: 10$.

Deposition of a ZnS shell does not alter the general XRD pattern of the AIS QDs but results in a distinct shift of the reflections to larger angles (see inserts in Fig. 1b), that is typical for the incorporation of $\mathrm{Zn}^{2+}$ ions into the chalcopyrite AIS structure ${ }^{6,9}$. In particular, the largest XRD peak shifts from $26.9^{\circ}$ to $27.4^{\circ}$ for fraction \#4 (left insert) and to $27.7-27.8^{\circ}$ for fraction $\# 9$ (right insert), the fact indicating that more $\mathrm{Zn}^{2+}$ ions can penetrate into the lattice of smaller AIS QDs.

XPS study of the size-selected QDs. The indium to silver ratio in the crude QD colloid synthesized at $(\mathrm{Ag}: \operatorname{In}: \mathrm{S})_{0}=1: 7: 10$ was expectably close to $7-8$, however, it decreased to $\sim 2$ after the sizeselective precipitation (Fig. 2) implying that In-MAA complexes were only partially converted into QDs and were left in the liquid phase unreacted after the QD growth.

Some enrichment with silver was observed for the fractions \#2 and \#3 of AIS QDs (Fig. 2a) and in the fraction \#2 of the AIS/ZnS QD colloid (Fig. 2b). For the fractions \#4-9 a rather constant composition of the AIS QDs was revealed (Fig. 2a). Similarly, a reasonably constant QD composition was also observed for the AIS/ZnS QDs in fractions \#3-9 (Fig. 2b). 
We can conclude, therefore, that in a broad range of fractions the precipitation/re-dispersion procedure does allow us to produce size-selected QD series differing considerably in the PL properties but having almost the same chemical composition.

As the QD amount in fractions \#10 and \#11 was very low we failed to obtain reproducible XPS results in the case of AIS QDs and found a somewhat lower $\mathrm{Zn}$ to Ag ratio than in fractions \#3-\#9 of AIS/ZnS QDs (Fig. 2b). A more or less stable value of the In to Ag ratio was also observed by XPS for other compositions of AIS QDs, in particular for the silver-rich QDs with (Ag:In:S) 0 = 2:7:10 (SI, Fig. S7a) and for sulfur-poor QDs prepared at (Ag:In:S $)_{0}=1: 7: 5$ (Fig. S7b).

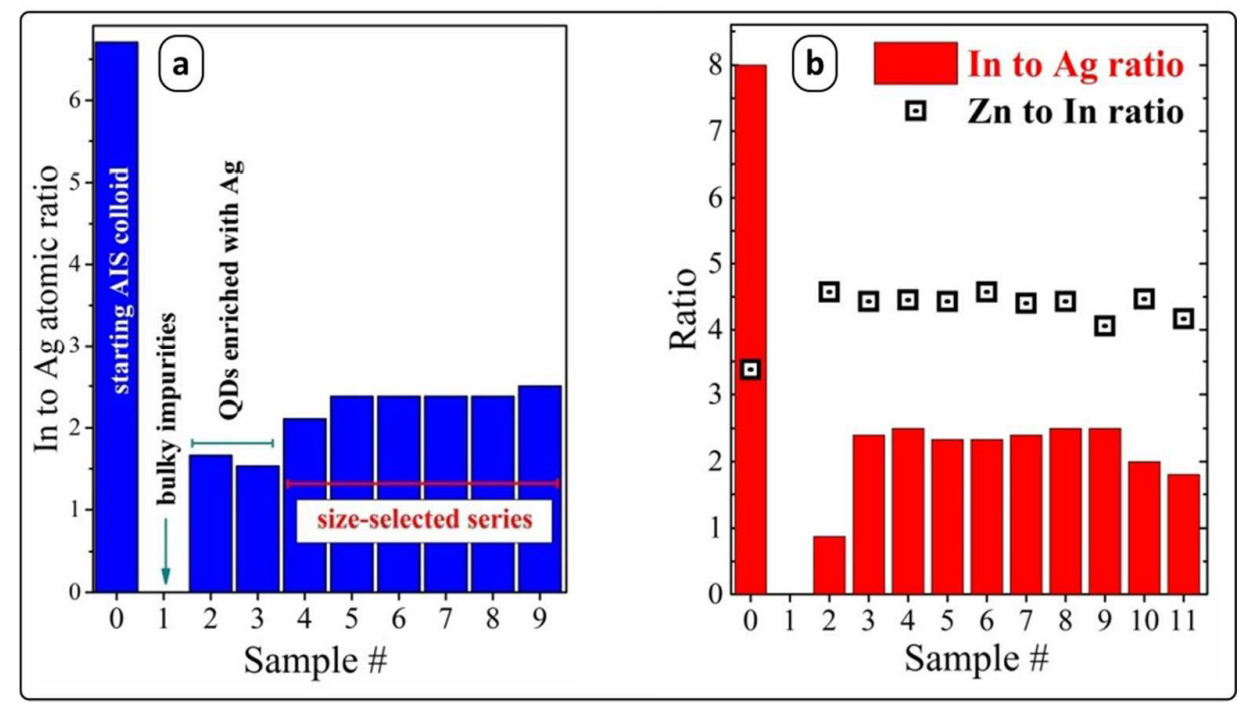

Figure 2. In-to-Ag $(\mathrm{a}, \mathrm{b})$ and Zn-to-In (b) atomic ratio determined by survey XPS analysis for AIS (a) and AIS/ZnS QDs (b). QDs were synthesized using an atomic ratio Ag:In:S of 1:7:10.

In the case of $\mathrm{AIS} / \mathrm{ZnS}$ QDs the $\mathrm{Zn}$ to $\mathrm{Ag}$ ratio was also found to be rather constant, around 4.5 for all studied QD fractions (Fig. 2b). Similarly to the In to Ag ratio, the $\mathrm{Zn}$ to Ag ratio detected in the QD samples differs from the nominal ratio $(\mathrm{Zn}: \mathrm{Ag})_{0}$ as well, indicating that a portion of Zn-MAA complex remained unreacted and is removed with the liquid phase during the size-selective precipitation of the AIS/ZnS QDs.

High-resolution XPS showed all the constituents of AIS and AIS/ZnS QDs to be in the expected valence states $-\mathrm{Ag}^{+}, \mathrm{In}^{3+}, \mathrm{Zn}^{2+}$, and $\mathrm{S}^{2-}$ for each studied fraction of the size-selected QDs. A detailed description of the high-resolution spectra deconvolution can be found in SI.

TEM of the size-selected QDs. Upon deposition on TEM grids, the AIS and AIS/ZnS QDs experience strong aggregation impeding the observation of discrete QDs, most probably due to the strong capillary forces in the drying drop of aqueous colloidal solution. To exclude these effects we transferred the QDs into toluene with an additional transfer surfactant ${ }^{50}$ and managed to deposit a 
relatively uniform layer of the QDs as shown in Fig. 3a. The QDs showed normal size distributions with a maximum at 3-3.5 $\mathrm{nm}$ for fractions \#3 and 4 and at around $2 \mathrm{~nm}$ for fractions \#8-10. The QDs synthesized at different nominal $(\mathrm{Ag}: \mathrm{In}: \mathrm{S})_{0}$ ratios revealed generally similar size distributions for the same QD fractions (compare Fig. 3c and 3d).

High-resolution TEM (HRTEM) images of MAA-capped AIS/ZnS are typically blurry, most probably due to an interference of organic ligands and zinc sulfide shells, but allow to distinguish separate 3-4-nm AIS QDs showing crystal fringes (Fig. 3b). The lattice period is $\sim 0.34 \mathrm{~nm}$ typical for the (002) face of chalcopyrite silver indium sulfide and differing notably from other possible binary phases, such as silver sulfide or indium sulfide ${ }^{12,20,22}$.
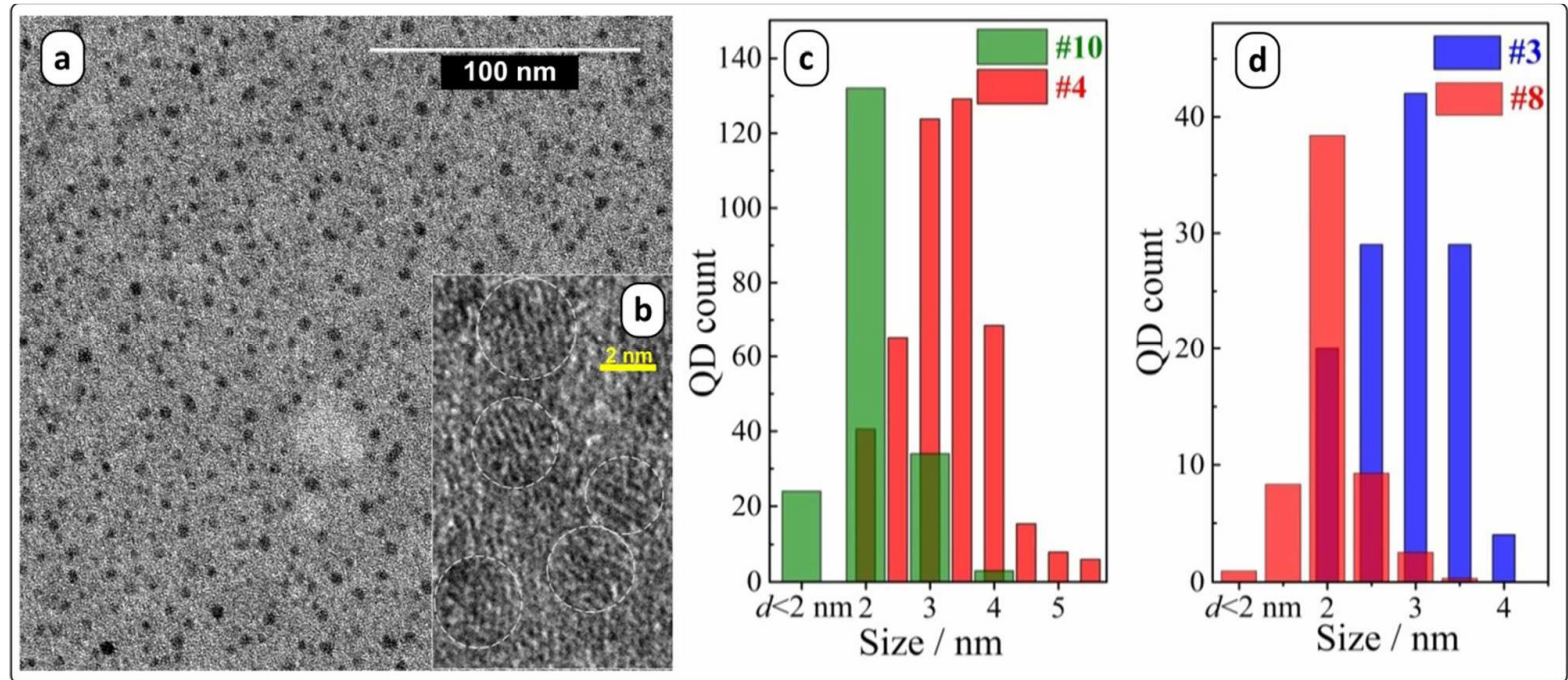

Figure 3. (a,b) TEM (a) and HRTEM (b) images of AIS/ZnS QDs in fraction \#4 synthesized at $(A g: I n: S)_{0}=1: 7: 10$. (c,d) Size distribution of AIS/ZnS QDs obtained using (Ag:In:S) $)_{0}=1: 7: 10$ (c) and 1:7:5 (d) based on the TEM data for fractions $\# 4$ and \#10 (c), \#3 and \#8 (d).

The TEM results agree well with the above-discussed size estimations from the XRD patterns indicating that we, indeed, operate in a narrow QD size range varying from around $2 \mathrm{~nm}$ to $3.5-4 \mathrm{~nm}$. In this view the size-selective precipitation is a unique method because it allows us to separate QD ensembles with a very narrow size distribution and to produce numerous fractions in a comparatively narrow size range. For example, the size-selective separation of $\mathrm{CdSe} / \mathrm{ZnS}$ QDs by introducing supercritical $\mathrm{CO}_{2}$ into hexane QD solutions yielded a series of fractions with distinctly different optical properties in a size range of $1.9-3.2 \mathrm{~nm}^{29}$. In a system similar to the one studied in the present paper the mercaptoacid-capped water-soluble CdSe QDs were separated by using 2-propanol into fractions encompassing a size range of 2.1-3.2 $\mathrm{nm}^{30}$. Dodecanthiol-capped CIS QDs were separated into 8-9 different fractions varying in the average QD size from 1.4 to $3 \mathrm{~nm}$ as proven by a combined study using TEM, XRD, and analytical ultracentrifugation ${ }^{44}$. A size-selective separation of liophylic $\operatorname{AgIn}(\mathrm{Ga}) \mathrm{S}_{2}$ QDs was successfully performed for 3-5 $\mathrm{nm}$ QDs $^{46}$. Probably, the most striking examples 
of a precise size-selective precipitation of QDs were reported for CdSe "magic-size" clusters that were separated into three distinct fractions in a size range of $1.5-2.0 \mathrm{~nm}^{32}$, for $1.8-3.5-\mathrm{nm}$ CdTe QDs separated into 24 distinctly resolved fractions by ultra-centrifugation ${ }^{39}$, as well as for the allylbenzenecapped Si QDs with 11 different fractions varying in the QD size from around 1 to $2 \mathrm{~nm}^{36}$.

Probing the AIS and AIS/ZnS QDs with Raman spectroscopy showed typical signs of tetragonal chalcopyrite structure with a relatively high degree of disorder. Also, the formation of core/shell AIS/ZnS structure, rather than homogeneous alloying between AIS and ZnS was deduced. The details of Raman spectra analysis can be found in SI.

Absorption spectra of the size-selected AIS QDs. The size-selected AIS QDs are characterized by a broad absorption band with the intensity lowering gradually to larger wavelengths and an edge at around 500-600 nm depending on the QD fraction (Fig. 4a). The integral absorbance of different fractions of size-selected AIS (AIS/ZnS) QDs is a direct measure of the molar AIS concentration in each fraction and was used to derive the quantitative distribution of the QDs in various fractions like the one presented in Fig. 1a (upper panel).

AIS is reported to be a direct-bandgap semiconductor near the absorption threshold, but the sizeselected AIS QDs reveal a quite smeared absorption band edge making a direct determination of the band gap $E_{\mathrm{g}}$ from the absorption spectra quite tricky, though this method is nevertheless used in some reports on size-selected AIS QD series ${ }^{14,51}$. A more precise $E_{\mathrm{g}}$ determination becomes possible after replotting the absorption spectra in the coordinates of the Tauc equation for direct allowed electron transitions $D_{\text {norm }}=$ const $\times\left(h v-E_{\mathrm{g}}\right) / h v^{52}$, using the normalized optical density $D_{\text {norm }}$ of the sizeselected QD fractions as an equivalent of the linear absorption coefficient (see insert in Fig. 4a).

Such plots show an extended linear section in the energy range of $2-3 \mathrm{eV}$ that can be approximated to $y=0$ yielding direct $E_{\mathrm{g}}$ values for each fraction. The bandgap of the size-selected AIS QDs (denoted as $E_{\mathrm{g}}{ }_{\text {in }}$ Table 1) was found to increase with an increment in the fraction number. In particular, for the typical synthesis at $(\mathrm{Ag}: \mathrm{In}: \mathrm{S})_{0}=1: 7: 10$ the band gap increases from $2.12 \mathrm{eV}$ for fraction \#3 to $2.30 \mathrm{eV}$ for fraction $\# 10$.

The $E_{\mathrm{g}}{ }^{d}$ increment of $0.18 \mathrm{eV}$ seems rather small for the QD size range of 2-3.5 $\mathrm{nm}$ probed in the present paper. A search for size scaling dependences of the optical properties of AIS QDs revealed a lack of reliable data on the "size $-E_{\mathrm{g}}$ " correlations for this ternary semiconductor, opposite to the broadly studied binary cadmium and lead chalcogenides and even for the closest analog of AIS$\mathrm{CuInS}_{2}$ QDs ${ }^{1,5,7,9}$. Even the electron and hole effective masses $\left(m^{*}\right.$ and $m^{*}$, respectively) are rarely reported for AIS compounds and the values of $m_{\mathrm{e}}{ }_{\mathrm{e}}=0.15 m_{0}$ and $m_{\mathrm{h}}{ }_{\mathrm{h}}=1.36 m_{0}$ for tetragonal AIS ${ }^{53}$ and $m_{\mathrm{e}}^{*}=\sim 0.15 m_{0}$ and $m_{\mathrm{h}}^{*}=\sim 1.9 m_{0}$ - for orthorhombic AIS ${ }^{54}$ can be found. Nevertheless, the calibration curve produced using the above $m_{\mathrm{e}}{ }_{\mathrm{e}}$ and $\mathrm{m}_{\mathrm{h}}{ }_{\mathrm{h}}$ values (SI, Fig. S11, curve 1) apparently does not provide a good correspondence to the reported experimental data. In view of this scarcity, possible changes of 
the electronic properties of AIS QDs induced by their size variations are typically evaluated using the charge effective masses reported for the closest AIS analogs, such as $\mathrm{CuInS}_{2}{ }^{14}$ or $\mathrm{AgInSe}_{2}{ }^{51}$.

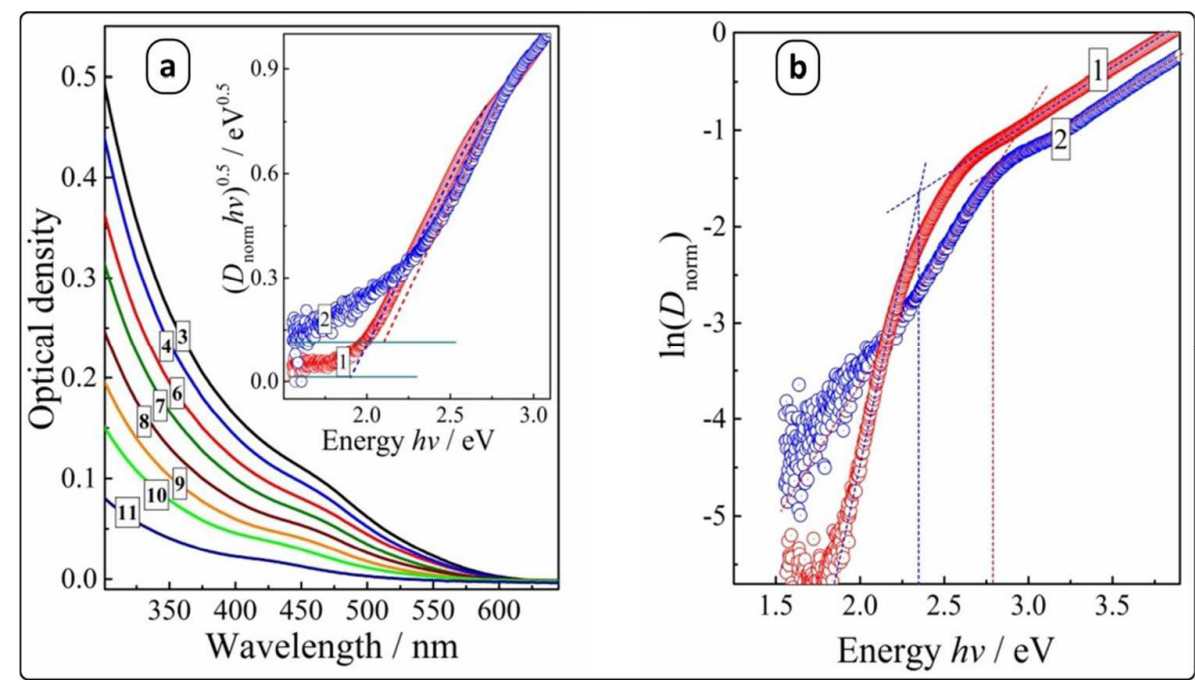

Figure 4. (a) Absorption spectra of size-selected AIS QDs. Numbers in the figure correspond to the fraction numbers. Insert: absorption spectra of fractions \#3 and \#11 in coordinates $\left(D_{\text {norm }} \times h v\right)^{0.5}$ vs. $h v$, where $D_{\text {norm }}$ - normalized optical density, $h v$ - quantum energy. (b) Absorption spectra of fractions $\# 3$ and \#11 in coordinates $\ln \left(D_{\text {norm }}\right)$ vs. $h v$.

Also, the calibration curves derived from semi-empirical calculations for CIS can be used for AIS as a first approximation because of the closeness in the effective charge carrier masses of the CIS an AIS compounds. For example, the "size $-E_{\mathrm{g}}$ " calibration curve produced for $\mathrm{CuInS}_{2}$ using a finite depth potential well model ${ }^{19}$ can be adapted to AIS by adding the calculated size-dependent increment to the bulk band gap of $\mathrm{AgInS}_{2}, E_{\mathrm{g}}{ }^{\text {bulk }}=1.85 \mathrm{eV}$ (the reported bulk band gap values for chalcopyrite AIS vary from $1.83 \mathrm{eV}^{53}$ to $1.87 \mathrm{eV}^{51}$ ), or other AIS polymorphs, such as $\mathrm{AgIn}_{5} \mathrm{~S}_{8}, E_{\mathrm{g}}$ bulk $=1.76 \mathrm{eV}^{55}$ (SI, Fig. S11). The calibration curves for both AIS polymorphs are very close to each other indicating the applicability to AIS QDs with a slightly varied composition and correspond reasonably well to the reported experimental data on the size dependences of the band gap of AIS QDs.

The estimations based on such curves showed that we should expect $E_{\mathrm{g}}=2.6-2.7 \mathrm{eV}$ for $2 \mathrm{~nm}$ AIS QDs and $E_{\mathrm{g}}=2.2-2.3 \mathrm{eV}$ for the $3 \mathrm{~nm}$ QDs indicating that the band gaps determined from the absorption spectra of the size-selected AIS QDs are lowered for some reasons. Also, the band gaps of the first fractions of the size-selected AIS QDs obtained at other (Ag:In:S) ratios, e.g. 2:7:10 (SI, Table S1) and 1:10:5 (SI, Table S2) were found to be even lower than the band gap of bulk tetragonal AIS. Band gaps lower than the bulk $E_{\mathrm{g}}$ of tetragonal AIS were also recently reported for ligand-free AIS QDs ${ }^{28}$. A thermal treatment of such AIS colloids performed to increase the size of the AIS QDs

did not result in the expected decrease in the band gap but contrary in its increase. This fact was explained by a high density of states near the band edges of AIS QDs due to abundant structural 
defects in the lattice and on the surface ${ }^{22}$. The presence of such states results in quite intense and long "tails" in the wavelength range of the absorption spectra of AIS QDs that mask the real absorption edge and contribute to a decrease of the measured $E_{\mathrm{g}}$ values. We believe that a similar situation is observed also for the present size-selected AIS QDs.

Table 1. Band gap determined by different methods - from the Tauc plot $\left(E_{\mathrm{g}}\right)^{d}$, by the derivative method $\left(E_{\mathrm{g}}{ }^{\mathrm{d}}\right.$, , and using the Urbach "tail" $\left(E_{\mathrm{g}}\right)$, Urbach energy $E_{\mathrm{U}}$, energy of the PL band maximum $E_{\mathrm{PL}}$, and the FWHM (full width at half maximum) of the PL band of size-selected AIS QDs. The original crude colloid was synthesized at $(\mathrm{Ag}: \operatorname{In}: \mathrm{S})_{0}=1: 7: 10$.

\begin{tabular}{|c|c|c|c|c|c|c|}
\hline Fraction \# & $E_{\mathrm{g}}{ }^{u}, \mathrm{eV}$ & $E_{\mathrm{g}}{ }^{\mathrm{ucr}}, \mathrm{eV}$ & $E_{\mathrm{g}}{ }^{\mathrm{N}}, \mathrm{eV}$ & $E_{\mathrm{U}}, \mathrm{meV}$ & $E_{\mathrm{PL}}, \mathrm{eV}$ & FWHM, eV \\
\hline 3 & 2.12 & 2.55 & 2.33 & 110 & 1.74 & 0.33 \\
\hline 4 & 2.14 & 2.54 & 2.35 & 120 & 1.75 & 0.34 \\
\hline 6 & 2.16 & 2.54 & 2.35 & 110 & 1.79 & 0.35 \\
\hline 7 & 2.19 & 2.55 & 2.37 & 120 & 1.81 & 0.36 \\
\hline 8 & 2.21 & 2.57 & 2.40 & 120 & 1.84 & 0.38 \\
\hline 9 & 2.24 & 2.60 & 2.56 & 200 & 1.86 & 0.40 \\
\hline 10 & 2.28 & 2.68 & 2.65 & 240 & 1.88 & 0.42 \\
\hline 11 & 2.30 & 2.73 & 2.79 & 360 & 1.93 & 0.45 \\
\hline
\end{tabular}

Note: accuracy of $E_{\mathrm{g}}{ }^{d}, E_{\mathrm{g}}{ }_{\mathrm{der}} E_{\mathrm{g}}, E_{\mathrm{U}}, E_{\mathrm{PL}}$, and FWHM determination is $\pm 0.005 \mathrm{eV}, \pm 0.02 \mathrm{eV}, \pm 0.01 \mathrm{eV}, \pm 10 \mathrm{meV}, \pm 0.01$ $\mathrm{meV}$, and $\pm 0.01 \mathrm{eV}$, respectively.

As can be seen from the insert in Fig. 4a, there exists a notable absorbance in the range of $h v<$ $E_{\mathrm{g}}$. As the absorption spectra of the colloidal AIS QDs registered in the broad spectral range of 350$1200 \mathrm{~nm}$ in the conventional transmission mode and by using an integrating sphere (SI, Fig. S12) almost coincide at $\lambda>500 \mathrm{~nm}$, a possible contribution of light scattering from the colloidal QDs into the absorbance at $h v<E_{\mathrm{g}}$ can be safely excluded.

A comparison of the spectra for fractions \#3 and \#11 shows that this absorbance increases strongly as the size of AIS QDs is reduced from 3-3.5 nm to $\sim 2 \mathrm{~nm}$. In the case of fraction \#11 the longer-wavelength "tail" extends as far as to $2.32-2.33 \mathrm{eV}$, that is above the band gap energy determined using the Tauc plot approach $(2.30 \mathrm{eV}$, Table 1). In this view, the deficiency of the conventional method for the determination of the band gap of such AIS QDs becomes evident and the effect of the defect-related "tail" states should be taken into account.

Earlier we reported on size-selected $\mathrm{ZnO} / \mathrm{SiO}_{2}$ QDs that exhibited quite broad absorption edges due to a broad distribution of the QD sizes ${ }^{56}$. To assess an average QD size in the ensemble we applied an approach of Pesika et al. ${ }^{57}$, where the mean size was determined from the position of an extremum 
of the first derivative of the absorption spectrum. We applied this method to the present AIS QDs expecting that this will mitigate the effect of the absorption tails. The first derivative absorption spectra indeed revealed a minimum (SI, Fig. S13) allowing for the determination of the average band gap of the AIS QDs in different fractions (denoted as $E_{\mathrm{g}}{ }^{\text {der }}$ in Table 1). The band gap derived by this approach is higher than $E_{\mathrm{g}}{ }^{d}$ and decreases from $2.73 \mathrm{eV}$ for fraction \#11 of the smallest QDs to $2.54-2.55 \mathrm{eV}$ for fractions \#3-\#7. However, the minima on the derivative spectra are quite broad and their amplitude decreases drastically as the fraction number increases from \#3-4 to \#9-11 (SI, Fig. S13) thus compromising the accuracy of the band gap determination. Moreover, the method is characterized by a very low sensitivity for the band gap variation for larger QDs in fractions \#3-7, and therefore its applicability is further limited.

Analysis of the Urbach dependence of size-selected AIS QDs. To make a correct distinction between the fundamental absorption caused by a direct electron transition from the "tail" absorption originating from the population of sub-bandgap states we applied the Urbach equation in the form $D_{\text {norm }}=$ const $\times \exp \left(\left(h v-E_{\mathrm{g}}\right) / E_{\mathrm{U}}\right)$, where $E_{\mathrm{U}}$ is the Urbach energy ${ }^{52}$. The absorption spectra of sizeselected AIS QDs presented as $\log \left(D_{\text {norm }}\right)$ versus $h v$ showed a high degree of linearity near the band edges (Fig. $4 \mathrm{~b}$ ) allowing $E_{\mathrm{U}}$ to be determined from the slope of the linear section of the spectral curve.

The value of Urbach energy depends on the nature of the semiconductor and on the degree of structural disorder in the crystal lattice ${ }^{52}$. Typical values reported for the metal chalcogenide nanoparticles vary in a broad range from tens to hundreds of $\mathrm{meV}^{1,3,52}$ reflecting the broad variety of possible defects in nanocrystalline semiconductors. The Urbach energy for the size-selected AIS QDs prepared at $(\mathrm{Ag}: \mathrm{In}: \mathrm{S})_{0}=1: 7: 10$ was found to depend distinctly on the QD size increasing from 110$120 \mathrm{meV}$ for fractions \#3,4 up to $360 \mathrm{meV}$ for fraction \#11 (Table 1). A strong variation of $E_{\mathrm{U}}$ with the QD size was also observed for other starting compositions (SI, Tables S1 and S2). The $E_{\mathrm{U}}$ increase clearly indicates a gradual disordering of the QD lattice as the QD size decreases, which is in accordance with the above-discussed XRD data. In this view, the $E_{\mathrm{U}}$ value can be accepted as a quantitative measure of the structural disorder of the QDs and the density of defect-related states in the band gap - the higher is $E_{\mathrm{U}}$ the broader is the distribution of sub-bandgap states and its effect on the absorption band edge.

As shown in Fig. 4b (insert) the upper limit of the Urbach absorption range is clearly visible in the spectrum of fraction $\# 11$ as a point where the slope of the linear section of the spectrum changes quite abruptly, around $2.8 \mathrm{eV}$. We suggest that this point can be approximately regarded as the real band gap of the AIS QDs, denoted as $E_{\mathrm{g}}^{\mathrm{U}}$ in Table 1. As the size of the particles increases (with decreasing fraction number) the slope of the linear Urbach section increases and an intermediate region can be observed between the linear Urbach section and a higher-energy part of the spectrum corresponding to the interband electron transitions. For example, this intermediate range extends from 
$\sim 2.2$ to around $2.5 \mathrm{eV}$ for the AIS QDs from fraction \#3 (Fig. 4b, insert, curve 1) and it precludes a precise determination of the intersection point between these two spectral features.

The highest $E_{\mathrm{g}}^{\mathrm{U}}, 2.79 \mathrm{eV}$, was found for the smallest QDs in fraction \#11 and it decreases gradually to $2.33-2.35$ for the fractions 3-4 with the largest QDs. These band gap values are of a rather arbitrary character but nevertheless, they reflect more correctly the band gap variation from fraction to fraction than the previously discussed $E_{\mathrm{g}}{ }^{d}$. In particular, both $E_{\mathrm{g}}^{\mathrm{U}}$ determined for fractions \#3 $(2.33 \mathrm{eV})$ and \#11 $(2.79 \mathrm{eV})$ fit the predictions of the "size - band gap" calibration curves for 3-3.5 $\mathrm{nm}$ and $2.0 \mathrm{~nm}$ AIS QDs much better than the corresponding $E_{\mathrm{g}}{ }^{d}$ values obtained from the Tauc method (SI, Fig. S11, brown diamonds). However, the $E_{\mathrm{g}}^{\mathrm{U}}$ values for fractions \#3-7, which are quite close due to the determination uncertainties, should be regarded as an approximation, while the real band gap resides somewhere between the $E_{\mathrm{g}}{ }^{d}$ and $E_{\mathrm{g}}{ }^{\mathrm{U}}$ in these cases.

In the case of AIS/ZnS QDs, no conclusive estimations can be done from the absorption spectra of the size-selected QDs because of the superposition of two phenomena: an increase of the band gap with reduced QD size and a band gap widening due to the doping with $\mathrm{Zn}$ (II). As a result of doping, the band gaps $E_{\mathrm{g}}{ }^{d}$ of AIS/ZnS QDs are in general by $\sim 0.5 \mathrm{eV}$ larger than the band gap of AIS QD core counterparts, increasing from $2.67 \mathrm{eV}$ for fraction \#3 to $2.90 \mathrm{eV}$ for fraction \#10 (SI, Table S3). Here also a linear Urbach section can be observed near the absorption band edge. The Urbach energy derived from the spectral dependence for the largest QDs, 150-170 meV, is somewhat higher than that for the core QDs. However, contrary to the core QDs, the core/shell AIS/ZnS QDs reveal no distinct dependence of $E_{\mathrm{U}}$ on the QD size (SI, Fig. Table S3), most probably due to the incorporation of $\mathrm{Zn}(\mathrm{II})$ ions into the core material resulting in a partial elimination of the structural defects.

PL properties of the size selected AIS and AIS/ZnS QDS. Similarly to the original crude AIS and AIS/ZnS colloids, the size-selected QDs revealed broad PL bands with a varied intensity and a band maximum shifting to higher wavelength as the fraction number increases (Fig. 5a). In general, the PL excitation (PLE) spectra of the size-selected AIS QDs and the corresponding absorption spectra are rather similar in shape and positions. The PLE band edge shows a red shift as the PL registration wavelength is increased (SI, Fig. S14) due to the QD size distribution in the fractionated colloids as revealed by the above-discussed TEM measurements. Similarly to the absorption spectra, the PLE spectra reveal sub-bandgap Urbach tails though of a comparatively lower intensity.

The PL band maximum of the AIS QDs, $E_{\mathrm{PL}}$, shifts gradually from $1.74 \mathrm{eV}$ for fraction \#3 to $1.93 \mathrm{eV}$ for fraction \#11 (Fig. 5a, Table 1) following the increase in the band gap of size-selected QDs. The shift can be clearly observed in the normalized PL spectra of the size-selected AIS QDs presented in SI (Fig. S15a). A shift, similar in the direction, but somewhat larger in magnitude, is also observed in the case of the size-selected AIS/ZnS QDs (SI, Table S3). 

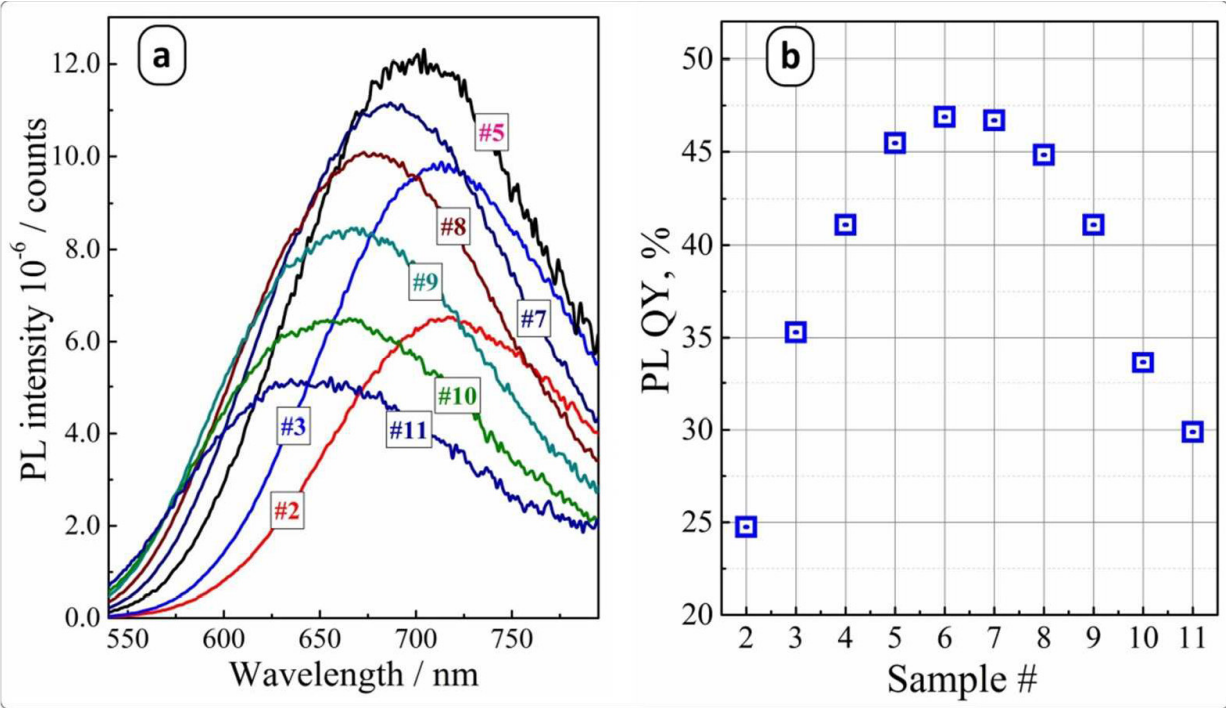

Figure 5. (a) PL spectra of size-selected AIS QDs (the numbers of the curves correspond to the QD fraction numbers). (b) PL QY of AIS/ZnS QDs from different samples. (Ag:In:S) $)_{0}=1: 7: 10$.

The upward shift of $E_{\mathrm{PL}}$ of the size-selected QDs is relatively small $-0.19 \mathrm{eV}$ for the core AIS QDs and $0.32 \mathrm{eV}$ in the case of the core/shell AIS/ZnS QDs, however, it results in a quite distinct change of the PL color. The PL color variation is especially pronounced for AIS/ZnS QDs (Fig. 1a, lower panel) which emit from deep red (fractions \#2,3) to bluish-green (fractions \#10,11). Such a rich variety of the emission color at so small variation of the QD size (from $\sim 2$ to $\sim 3-3.5 \mathrm{~nm}$ ) can arise from the fact that a small upward shift of the PL band energy results simultaneously in a gain of shorterwavelength emission and a loss in the longer-wavelength PL, apparently producing a strong net effect for the human eye. By varying the precursor ratio $(\mathrm{Ag}: \mathrm{In}: \mathrm{S})_{0}$, the PL hue can additionally be tuned both for size-selected AIS and AIS/ZnS QDs (SI, Fig. S16).

The PL intensity of both AIS and AIS/ZnS QDs increases from fraction \#2 to \#5, reaches a maximal value for fraction \#6 and decreases for higher fraction numbers. In the case of AIS/ZnS QDs $\left((\mathrm{Ag}: \mathrm{In}: \mathrm{S})_{0}=1: 7: 10,(\mathrm{Zn}: \mathrm{Ag})_{0}=10: 1\right)$ the PL QY grows from $\sim 25 \%$ for fraction $\# 2$ to the maximal value of $47 \%$ for fraction 6 and then decreases to 30\% for fraction \#11 (Fig. 5b). Expectedly, the crude colloid shows an intermediate PL QY value of $37 \pm 1 \%$, reflecting contributions of various fractions according to their population in the crude ensemble.

The dome-shaped dependences between the QD size and PL QY are common for size-selected series of semiconductor QDs produced by selective precipitation from QD ensembles ${ }^{38}$. The efficiency of radiative recombination typically increases with a QD size reduction due to a stronger confinement of the charge carriers and overlapping of their wavefunctions ${ }^{1,3,4,7}$. However, the probability of radiationless recombination increases as well, due an increase of the structural disorder and defect density in smaller QDs, as well as larger overlap of stronger confined wavefunctions with surface 
states. A counter-balancing of both trends results in an optimal intermediate QD size, at which the PL QY is maximal.

The PL band of both AIS and AIS/ZnS QDs are symmetrical and can be in all cases approximated by a single Gaussian profile (see examples for the AIS QDs from fractions \#3-\#9 in SI, Fig. S15b). The fitting of the PL spectra with Gaussian curves revealed that the spectral width (FWHM) of the PL band of the size-selected AIS QDs steadily grows from $0.33 \mathrm{eV}$ for the largest QDs in fraction \#3 to $0.45 \mathrm{eV}$ for fraction \#11 with the smallest QDs (Table 1). As the precipitation/redispersion procedure produces QD fractions with roughly the same size distribution, the PL band broadening should be accounted for by other reasons, in particular by an increase of the density (population) of possible defect midgap states participating in the electron-hole recombination.

As discussed above, the structural imperfection of the size-selected AIS QDs can be quantitatively characterized by the magnitude of the Urbach energy $E_{\mathrm{U}}$ derived from the absorption spectra. We found that the Urbach energy of the AIS QDs correlates distinctly with the spectral width of their PL band. Figure 6 shows a dependence between $E_{\mathrm{U}}$ and FWHM for the size-selected AIS QDs (blue circles) produced at different $(\mathrm{Ag}: \mathrm{In}: \mathrm{S})_{0}$ ratios and separated in different fractions. Both parameters reveal a clear correlation, where the PL bandwidth increases from around $0.33 \mathrm{eV}$ to $0.45 \mathrm{eV}$ with $E_{\mathrm{U}}$ variation from $90-100 \mathrm{meV}$ to around $350 \mathrm{meV}$. The Pearson correlation coefficient for $E_{\mathrm{U}}-\mathrm{FWHM}$ data set depicted in Fig. 6 is 0.94 (the maximum is 1.00).

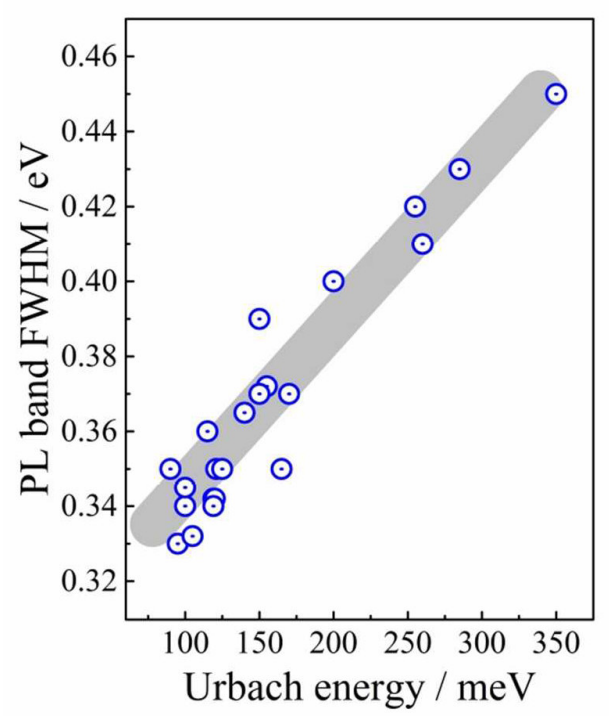

Figure 6. Thes spectral width of the PL band of the size-selected AIS QDs as a function of Urbach energy calculated from the corresponding absorption spectra. The gray bar serves only as an eye guide for the correlation between FWHM and $E_{\mathrm{U}}$.

The size-selected AIS/ZnS QDs, on the contrary, reveal no distinct variation of the FWHM with the QD size and fraction number (SI, Table S3). In view of the above-discussed $E_{\mathrm{U}}-\mathrm{FWHM}$ 
correlation this fact is expected because the AIS/ZnS QDs exhibited no appreciable variation of $E_{\mathrm{U}}$ as well. The $E_{\mathrm{U}}$ values for the core/shell AIS/ZnS QDs in the first fractions $(\# 3,4)$ are higher than in the corresponding fractions of AIS/QDs and so the observed PL band FWHM of AIS/ZnS in these fractions is also larger than that of the core AIS QDs (compare Table 1 and Table S3 in SI).

UV photoelectron spectroscopy of size-selected AIS and AIS/ZnS QDs. In view of possible applications of the AIS (AIS/ZnS) QDs not only as luminescing species but also as light absorbers in solar cells, we studied the size-selected QD series produced in this work with UPS to probe a possible size-dependence of the valence/conduction band positions. The UPS spectra were collected for sizeselected QDs deposited onto gold films and FTO substrates. Typical spectra obtained for AIS QDs from different fractions as well as for AIS/ZnS QDs can be found in SI (Fig. S17).

No distinct variation of the valence band top position as a function of the QD size (fraction number), the presence/absence of the $\mathrm{ZnS}$ shell, and the substrate type was observed. In particular, $E_{\mathrm{VB}}$ varies from 6.4 to $6.7 \mathrm{eV}$ for AIS QDs (SI, Table S4) with an average value at $6.6 \mathrm{eV}$ versus the vacuum level. The original crude AIS QDs showed somewhat deeper $E_{\mathrm{VB}}$ at $6.9 \mathrm{eV}$, most probably due to the presence of residual MA complexes on the QD surfaces, which are typically eliminated during the fractionation. The size-selected core/shell AIS/ZnS QDs revealed the same average $E_{\mathrm{VB}}$ position at $6.6 \mathrm{eV}$ (SI, Table S5), despite the expectations for the influence of the $\mathrm{ZnS}$ shell on the overall valence band position ${ }^{58}$. Similarly, the size-selected AIS QDs deposited onto FTO showed a very narrow spread of $E_{\mathrm{VB}}$ values from 6.9 to $7.1 \mathrm{eV}$ with an average value at $7.0 \mathrm{eV}$ (SI, Table S6). It should be mentioned that for $(\mathrm{AgIn})_{\mathrm{x}} \mathrm{Zn}_{2(1-\mathrm{x})} \mathrm{Se}_{2}$ a non-monotonous compositional dependence of the VBedge similar to our observations was predicted by calculations ${ }^{59}$.

As discussed above, the size-selected AIS QDs exhibit quite distinct dependences of the optical properties (both absorption and PL) on their size. Also, unintentional doping of the AIS core QDs with $\mathrm{Zn}$ (II) during the $\mathrm{ZnS}$ shell deposition alters their electronic properties irrespective of the core size and, therefore, we may expect both effects of the size and especially the $\mathrm{Zn}^{2+}$ doping to be observable in the UPS spectra. The absence of both such effects indicates that the properties of the QDs are considerably influenced by external factors, most probably by the interaction with the conductive support.

The effects of "pinning" of the valence band level as a result of electron interaction with supports were indeed reported for small metal chalcogenide QDs. In particular, CdTe QDs revealed no size dependence of the $E_{\mathrm{VB}}$ position in a range of 3.7-6 nm when deposited onto the gold surfaces modified with dithiolate ligands ${ }^{60}$. This effect was attributed to a strong interaction between the QD surface and the electron gas of the metal resulting in the pinning of the $E_{\mathrm{VB}}$ level to the Fermi level of gold. Similarly, pinning of the VB level was reported for CdSe QDs $(2.1-4.2 \mathrm{~nm})$ bonded to the surface of a $\mathrm{ZnO}$ single crystal via a short mercaptopropionate linker ${ }^{61}$. 
In the present case, the QDs can also interact both with gold and FTO via short surface mercaptoacetate ligands. Thus, a strong influence of the conductive substrate on the properties of adsorbed AIS QDs can be envisaged as well, resulting in the pinning of the $E_{\mathrm{VB}}$ level. This result, though discouraging from the viewpoint of the $E_{\mathrm{VB}}$ determination, can be potentially positive for the application of size-selected AIS QDs in solar cells because some degree of $E_{\mathrm{VB}}$ pinning can also be expected after the QD deposition onto wide-bandgap scaffolds, like $\mathrm{TiO}_{2}$ and $\mathrm{ZnO}$, typically used in solar cells of various types. As the band gap of AIS QDs will increase with the size decreasing, the entire $E_{\mathrm{g}}$ increment will be converted into an increment of the conduction band energy due to the $E_{\mathrm{VB}}$ pinning. As a result, we may expect a distinct size-dependence of the rate of charge transfer from the conduction band of photoexcited AIS QDs to the wide-bandgap collector. Further investigations of the possible pinning effects to other substrates are in progress in our group.

\section{Conclusions}

Size-selected series of water-soluble luminescent non-stoichiometric mercaptoacetate-stabilized silver indium sulfide (AIS) and core/shell AIS/ZnS QDs were produced by the precipitation technique. Deposition of a $\mathrm{ZnS}$ shell onto AIS cores prior to their fractionation was found to considerably improve the stability of the particles toward aggregation and oxidation as well as to increase the PL efficiency. Up to 10-11 fractions of size-selected AIS (AIS/ZnS) QDs were isolated differing distinctly in their optical properties and emitting in a broad color range from deep-red (fractions with larger QDs) through yellow-orange (fractions with intermediate sizes) to bluish-green (fractions with smaller sizes). The PL hue can be additionally varied by adjusting the composition of the starting crude AIS (AIS/ZnS) colloids. The PL quantum yield of size-selected core/shell AIS/ZnS QDs varied among the fractions reaching up to $47 \%$ for the intermediate (yellow-orange emitting) fractions.

The size range of the isolated AIS (AIS/ZnS) QDs was found to be quite narrow, varying from $\sim 2 \mathrm{~nm}$ for the smallest QDs to 3-4 $\mathrm{nm}$ for the largest QDs. The results of XRD and Raman spectroscopy provided arguments in favor of the formation of heterogeneous AIS core/ZnS shell QDs rather than a homogeneous alloying between Ag-In-S and ZnS, though a distinct doping of the AIS core with $\mathrm{Zn}^{2+}$ was deduced from the optical absorption and PL spectra. According to the results of Xray photoelectron spectroscopy, the AIS and AIS/ZnS QDs have roughly the same composition throughout this size range. A study of the size-selected AIS and AIS/ZnS QDs by UV photoelectron spectroscopy on Au and FTO substrates revealed a strong pinning of the energy of $E_{\mathrm{VB}}$ to the Fermi level of the conductive substrate making impossible an accurate $E_{\mathrm{VB}}$ determination. 
As shown by XRD, a decrease of the size of AIS (AIS/ZnS) QDs is accompanied with an increase of the structural imperfection/disorder in the QDs. The high degree of structural disorder of the size-selected QDs is also evidenced by notable Urbach absorption near and below the fundamental absorption band edge. In the case of AIS QDs, the Urbach energy was found to increase from 90-100 $\mathrm{meV} \mathrm{nm}$ for the largest QDs up to $350 \mathrm{meV}$ for the smallest QDs indicating a broadening of possible subbandgap (midgap) states participating in the sub- $E_{\mathrm{g}}$ light absorption.

A distinct correlation between the Urbach energy and the spectral width of the PL band of the size-selected AIS QDs was found, both parameters increasing considerably as the size of the AIS QDs is reduced from $3-4 \mathrm{~nm}$ to $\sim 2 \mathrm{~nm}$. This correlation unambiguously indicates the participation of structural defects in the radiative electron-hole recombination and the fact that the spectral distribution of the PL quanta is governed by a population of sub-bandgap states of AIS (AIS/ZnS) QDs.

The Supporting Information (SI): details on acquisition of XPS, UPS and Raman spectra; PL spectra of crude AIS colloids produced at various starting conditions; additional TEM data on fractioned AIS QDs; XRD patterns of AIS/ZnS QDs and individual silver and indium sulfide phases; XPS and Raman data for the size-selected QD series produced in different starting conditions; description of high-resolution XPS spectra and Raman spectra of the size-selected AIS and AIS/ZnS QDs; correlations between the size and band gap of AIS QDs; data on spectral (absorption and PL) parameters of the size-selected AIS and AIS/ZnS QDs; photographs of various size-selected AIS and AIS/ZnS colloids under UV illumination; UPS spectra and results of $E_{\mathrm{VB}}$ determination for the sizeselected AIS and AIS/ZnS QDs.

\section{Acknowledgements}

This work was funded by the European Union's Horizon 2020 research and innovation program under the Marie Skłodowska-Curie grant agreement No 701254 and by The Volkswagen Foundation (project "New functionalities of semiconductor nanocrystals by controllable coupling to molecules").

\section{References}


1. Kershaw, S. V.; Susha, A. S.; Rogach, A. L. Narrow Bandgap Colloidal Metal Chalcogenide Quantum Dots: Synthetic Methods, Heterostructures, Assemblies, Electronic and Infrared Optical Properties. Chem. Soc. Rev. 2013, 42, 3033-3087.

2. Wang, R.; Shang, Y.; Kanjanaboos, P.; Zhou, W.; Ning, Z.; Sargent, E. H. Colloidal Quantum Dot Ligand Engineering for High Performance Solar Cells. Energy Environ. Sci. 2016, 9, 1130-1143.

3. Pietryga, J. M., Park, Y. S.; Lim, J.; Fidler, A. F.; Bae, W. K.; Brovelli, S.; Klimov, V. I. Spectroscopic and Device Aspects of Nanocrystal Quantum Dots. Chem. Rev. 2016, 116, 1051310622.

4. Knowles, K. E.; Hartstein, K. H.; Kilburn, T. B.; Marchioro, A.; Nelson, H. D.; Whitham, P. J.; Gamelin, D. R. Luminescent Colloidal Semiconductor Nanocrystals Containing Copper: Synthesis, Photophysics, and Applications. Chem. Rev. 2016, 116, 10820-10851.

5. Zu, G.; Zeng, S.; Zhang, B.; Swihart, M. T.; Yong, K. T.; Prasad, P. N. New Generation CadmiumFree Quantum Dots for Biophotonics and Nanomedicine. Chem. Rev. 2016, 116, 12234-12327.

6. Fan, F. J.; Wu, L.; Yu, S. H. Energetic I-III-VI ${ }_{2}$ and $\mathrm{I}_{2}-\mathrm{II}-\mathrm{IV}-\mathrm{VI}_{4}$ Nanocrystals: Synthesis, Photovoltaic and Thermoelectric Applications. Energy Environ. Sci. 2014, 7, 190-208.

7. Kolny-Olesiak, J.; Weller, H. Synthesis and Application of Colloidal CuInS 2 Semiconductor Nanocrystals. ACS Appl. Mater. Interfaces 2013, 5, 12221-12237.

8. Zheng, Z.; Ji, H.; Yu, P.; Wang, Z. Recent Progress Towards Quantum Dot Solar Cells with Enhanced Optical Absorption. Nanoscale Res. Lett. 2016, 11, 266-274.

9. Aldakov, D.; Lefrançois, A.; Reiss, P. Ternary and Quaternary Metal Chalcogenide Nanocrystals: Synthesis, Properties and Applications. J. Mater. Chem. C 2013, 1, 3756-3776.

10. Thomas, S. R.; Chen, C. W.; Date, M.; Wang, Y. C.; Tsai, H. W.; Wang, Z. M.; Chueh, Y. L. Recent Developments in The Synthesis of Nanostructured Chalcopyrite Materials and Their Applications: A Review. RSC Adv. 2016, 6, 60643-60656.

11. Sharma, D.; Jha, R.; Kumar, S. Quantum Dot Sensitized Solar Cells: Recent Advances and Future Perspectives in Photoanode. Sol. En. Mater. \& Sol. Cells 2016, 155, 294-322.

12. Hamanaka, Y.; Ogawa, T.; Tsuzuki, M.; Kuzuya, T. Photoluminescence Properties and Its Origin of $\mathrm{AgInS}_{2}$ Quantum Dots with Chalcopyrite Structure. J. Phys. Chem. C 2011, 115, 1786-1792.

13. Jara, D. H.; Yoon, S. J.; Stamplecoskie, K. G.; Kamat, P.V. Size-Dependent Photovoltaic Performance of $\mathrm{CuInS}_{2}$ Quantum Dot-Sensitized Solar Cells. Chem. Mater. 2014, 26, 7221-7228.

14. Torimoto, T.; Tada, M.; Dai, M.; Kameyama, T.; Suzuki, S.; Kuwabata, S. Tunable Photoelectrochemical Properties of Chalcopyrite $\operatorname{AgInS}_{2}$ Nanoparticles Size-Controlled with a Photoetching Technique. J. Phys. Chem. C 2012, 116, 21895-21902.

15. Zhong, H.; Lo, S. S.; Mirkovic, T.; Li, Y.; Ding, Y.; Li, Y.; Scholes, G. D. Noninjection GramScale Synthesis of Monodisperse Pyramidal $\mathrm{CuInS}_{2}$ Nanocrystals and Their Size-Dependent 
Properties. ACS Nano 2010, 4, 5253-5262.

16. Zhong, H.; Wang, Z.; Bovero, E.; Lu, Z.; von Veggel, F. C. J. M.; Scholes, G. D. Colloidal $\mathrm{CuInSe}_{2}$ Nanocrystals in the Quantum Confinement Regime: Synthesis, Optical Properties, and Electroluminescence. J. Phys. Chem. C 2011, 115, 12393-12402.

17. Panthani, M. G.; Stolle, C. J.; Reid, D. K.; Rhee, D. J.; Harvey, T. B.; Akhavan, V. A.; Yu, Y.; Korgel, B. A. CuInSe 2 Quantum Dot Solar Cells with High Open-Circuit Voltage. J. Phys. Chem. Lett. 2013, 4, 2030-2034.

18. Li, T. L.; Teng, H. Solution Synthesis of High-Quality CuInS ${ }_{2}$ Quantum Dots as Sensitizers for $\mathrm{TiO}_{2}$ Electrodes. J. Mater. Chem. 2010, 20, 3656-3664.

19. Omata, T.; Nose, K.; Otsuka-Yao-Matsuo, S. Size Dependent Optical Band Gap of Ternary I-III$\mathrm{VI}_{2}$ Semiconductor Nanocrystals. J. Appl. Phys. 2009, 105, 073106.

20. Torimoto, T.; Kameyama, T.; Kuwabata, S. Photofunctional Materials fabricated with Chalcopyrite-Type Semiconductor Nanoparticles Composed of $\mathrm{AgInS}_{2}$ and Its Solid Solutions. $J$.

Phys. Chem. Lett. 2014, 5, 336-347.

21. Luo, Z.; Zhang, H.; Huang, J.; Zhong, X. One-Step Synthesis of Water-Soluble AgInS 2 and ZnS$\mathrm{AgInS}_{2}$ Composite Nanocrystals and Their Photocatalytic Activities. J. Colloid Interface Sci. 2012, $377,27-33$.

22. Kadlag, K. P.; Patil, P.; Rao, M. J.; Datta, S.; Nag, A. Luminescence and Solar Cell from LigandFree Colloidal AgInS 2 Nanocrystals. CrystEngComm 2014, 16, 3605-3612.

23. Regulacio, M. D.; Win, K. Y.; Lo, S. L.; Zhang, S. Y.; Zhang, X.; Wang, S.; Han, M. Y.; Zheng, Y. Aqueous Synthesis of Highly Luminescent $\operatorname{AgInS}_{2}-\mathrm{ZnS}$ Quantum Dots and Their Biological Applications. Nanoscale 2013, 5, 2322-2327.

24. Raevskaya, A. E.; Ivanchenko, M. V.; Stroyuk, O. L.; Kuchmiy, S. Y.; Plyusnin, V. F. Luminescent Ag-doped $\operatorname{In}_{2} \mathrm{~S}_{3}$ Nanoparticles Stabilized by Mercaptoacetate in Water and Glycerol. J. Nanopart. Res. 2015, 17, 135-143.

25. Raevskaya, A.; Rosovik, O.; Kozytskiy, A.; Stroyuk, O.; Dzhagan, V.; Zahn, D. NonStoichiometric Cu-In-S@ZnS Nanoparticles Produced in Aqueous Solutions as Light Harvesters for Liquid-Junction Photoelectrochemical Solar Cells. RSC Adv. 2016, 6, 100145-100157.

26. Chemseddine, A.; Weller H. Highly Monodisperse Quantum Sized CdS Particles by Size Selective Precipitation. Ber. Bunsenges. Phys. Chem. 1993, 97, 636-638.

27. Murray, C. B.; Norris, D. J.; Bawendi, M. G. Synthesis and Characterization of Nearly Monodisperse CdE (E $=\mathrm{S}$, Se, Te) Semiconductor Nanocrystallites. J. Am. Chem. Soc. 1993, 115, 8706-8715.

28. Wang, C. L.; Fang, M.; Xu, S. H.; Cui, Y. P. Salts-Based Size-Selective Precipitation: Toward Mass Precipitation of Aqueous Nanoparticles. Langmuir 2010, 26, 633-638. 
29. Anand, M.; Odom, L. A.; Roberts, C. B. Finely Controlled Size-Selective Precipitation and Separation of $\mathrm{CdSe} / \mathrm{ZnS}$ Semiconductor Nanocrystals Using $\mathrm{CO}_{2}$-Gas-Expanded Liquids. Langmuir 2007, 23, 7338-7343.

30. Rogach, A. L.; Kornowski, A.; Gao, M.; Eychmüller, A.; Weller, H. Synthesis and Characterization of a Size Series of Extremely Small Thiol-Stabilized CdSe Nanocrystals. J. Phys. Chem. B 1999, 103, 3065-3069.

31. Rogach, A. L.; Talapin, D. V.; Shevchenko, E. V.; Kornowski, A.; Haase, M.; Weller, H. Organization of Matter on Different Size Scales: Monodisperse Nanocrystals and Their Superstructures. Adv. Funct. Mater. 2002, 12, 653-664.

32. Kudera, S.; Zanella, M.; Giannini, C.; Rizzo, A.; Li, Y.; Gigli, G.; Cingolani, R.; Ciccarella, G.; Spahl, W.; Parak, W. J.; Manna, L. Sequential Growth of Magic-Size CdSe Nanocrystals. Adv. Mater. 2007, 19, 548-552.

33. Talapin, D. V.; Shevchenko, E. V.; Kornowski, A.; Gaponik, N.; Haase, M.; Rogach, A. L.; Weller, H. A New Approach to Crystallization of CdSe Nanoparticles into Ordered ThreeDimensional Superlattices. Adv. Mater. 2001, 13, 1868-1871.

34. Holmes, M. A.; Townsend, T. K.; Osterloh, F. E. Quantum Confinement Controlled Photocatalytic Water Splitting by Suspended CdSe Nanocrystals. Chem. Commun. 2012, 48, 371-373.

35. Bao, H.; Wang, E.; Dong, S. One-Pot Synthesis of CdTe Nanocrystals and Shape Control of Luminescent CdTe-Cystine Nanocomposites. Small 2006, 2, 476-480.

36. Gaponik, N.; Talapin, D. V.; Rogach, A. L.; Hoppe, K.; Shevchenko, E. V.; Kornowski, A.; Eychmüller, A.; Weller, H. Thiol-Capping of CdTe Nanocrystals: An Alternative to Organometallic Synthetic Routes. J. Phys. Chem. B 2002, 106, 7177-7185.

37. Lesnyak, V.; Gaponik, N.; Eychmüller, A. Colloidal Semiconductor Nanocrystals: The Aqueous Approach. Chem. Soc. Rev. 2013, 42, 2905-2929.

38. Talapin, D. V.; Rogach, A. L.; Shevchenko, E. V.; Kornowski, A.; Haase, M.; Weller, H. Dynamic Distribution of Growth Rates within the Ensembles of Colloidal II-VI and III-V Semiconductor Nanocrystals as a Factor Governing Their Photoluminescence Efficiency. J. Am. Chem. Soc. 2002, 124, 5782-5790.

39. Karabudak, E.; Brookes, E.; Lesnyak, V.; Gaponik, N.; Eychmüller, A.; Walter, J.; Segets, D.; Peukert, W.; Wohlleben, W.; Demeler, B.; Cölfen, H. Simultaneous Identification of Spectral Properties and Sizes of Multiple Particles in Solution with Subnanometer Resolution. Angew. Chem. Int. Ed. 2016, 55, 11770-11774.

40. Mastronardi, M. L.; Maier-Flaig, F.; Faulkner, D.; Henderson, E. J.; Kübel, C.; Lemmer, U.; Ozin, G. A. Size-Dependent Absolute Quantum Yields for Size-Separated Colloidally-Stable Silicon Nanocrystals. Nano Lett. 2012, 12, 337-342. 
41. Guzelian, A. A.; Katari, J. E. B.; Kadavanich, A. V.; Banin, U.; Hamad, K.; Juban, E.; Alivisatos, A. P.; Wolters, R. H.; Arnold, C. C.; Heath, J. R. Synthesis of Size-Selected, Surface-Passivated InP Nanocrystals, J. Phys. Chem. 1996, 100, 7212-7219.

42. Nam, D. E.; Song, W. S.; Yang, H. Facile, Air-Insensitive Solvothermal Synthesis of EmissionTunable $\mathrm{CuInS}_{2} / \mathrm{ZnS}$ Quantum Dots with High Quantum Yields. J. Mater. Chem. 2011, 21, 1822018226.

43. Xie, R.; Rutherford, M.; Peng, X. Formation of High-Quality I-III-VI Semiconductor Nanocrystals by Tuning Relative Reactivity of Cationic Precursors. J. Am. Chem. Soc. 2009, 131, 5691-5697.

44. Akdas, T.; Walter, J.; Segets, D.; Distaso, M.; Winter, B.; Birajar, B.; Spieckler, E.; Peukert, W. Investigation of The Size-Property Relationship in $\mathrm{CuInS}_{2}$ Quantum Dots. Nanoscale 2015, 7, 18105 18118.

45. Chang, S. H.; Chiu, B. C.; Gao, T. L.; Jheng, S. L.; Tuan, H. Y. Selective synthesis of copper gallium sulfide $\left(\mathrm{CuGaS}_{2}\right)$ nanostructures of different sizes, crystal phases, and morphologies. CrystEngComm 2014, 16, 3323-3330.

46. Uematsu, T.; Doi, T.; Torimoto, T.; Kuwabata, S. Preparation of Luminescent AgInS ${ }_{2}-\mathrm{AgGaS}_{2}$ Solid Solution Nanoparticles and Their Optical Properties. J. Phys. Chem. Lett. 2010, 1, 3283-3287.

47. Collord, A. D.; Hillhouse, H. W. Composition Control and Formation Pathway of CZTS and CZTGS Nanocrystal Inks for Kesterite Solar Cells. Chem. Mater. 2015, 27, 1855-1862.

48. Singh, A.; Geaney, H.; Laffir, F.; Ryan, K.M. Colloidal Synthesis of Wurtzite $\mathrm{Cu}_{2} \mathrm{ZnSnS}_{4}$ Nanorods and Their Perpendicular Assembly. J. Am. Chem. Soc. 2012, 134, 2910-2913.

49. Raevskaya, A. E.; Ivanchenko, M. V.; Skoryk, M. A.; Stroyuk, O. Brightly Luminescent Colloidal Ag-In-S Nanoparticles Stabilized in Aqueous Solutions by Branched Polyethyleneimine. J. Lumin. 2016, 178, 295-300.

50. Zhang, H.; Cui, Z.; Wang, Y.; Zhang K.; Ji, X.; Lü, C.; Yang, B.; Gao, M. From Water-Soluble CdTe Nanocrystals to Fluorescent Nanocrystal-Polymer Transparent Composites Using Polymerizable Surfactants. Adv. Mater. 2003, 15, 777-780.

51. Hamanaka, Y.; Ogawa, T.; Tsuzuki, M. Photoluminescence Properties and Its Origin of $\operatorname{AgInS}_{2}$ Quantum Dots with Chalcopyrite Structure. J. Phys. Chem. C 2011, 115, 1786-1792.

52. Gaponenko, S. V. Optical Properties of Semiconductor Nanocrystals; Cambridge University Press: Cambridge, U.K., 1998.

53. Huang, D.; Persson, C. Photocatalyst $\operatorname{AgInS}{ }_{2}$ for Active Overall Water-Splitting: A First-Principles Study. Chem. Phys. Lett. 2014, 591, 189-192.

54. Liu, J.; Chen, S.; Liu, Q.; Zhu, Y.; Lu, Y. Density Functional Theory Study on Electronic and Photocatalytic Properties of Orthorhombic AgInS 2 . Comput. Mater. Sci. 2014, 91, 159-164.

55. Li, K.; Chai, B.; Peng, T.; Mao, J.; Zan, L. Preparation of $\mathrm{AgIn}_{5} \mathrm{~S}_{8} / \mathrm{TiO}_{2}$ Heterojunction Nanocom- 
posite and Its Enhanced Photocatalytic $\mathrm{H}_{2}$ Production Property under Visible Light. ACS Catal. 2013, 3, 170-177.

56. Raevskaya, A. E.; Panasiuk, Y. V.; Stroyuk, O. L.; Kuchmiy, S.Y.; Dzhagan, V. M.; Milekhin, A. G.; Yeryukov, N. A.; Sveshnikova, L. A.; Rodyakina, E. E.; Plyusnin, V. F.; Zahn, D. R. T. Spectral and Luminescent Properties of $\mathrm{ZnO} / \mathrm{SiO}_{2}$ Core/Shell Nanoparticles with Size-Selected $\mathrm{ZnO}$ Cores. $R S C A d v$. 2014, 4, 63393-63401.

57. Pesika, N. S.; Stebe, K. J.; Searson, P. C. Relationship between Absorbance Spectra and Particle Size Distributions for Quantum-Sized Nanocrystals. J. Phys. Chem. B 2003, 107, 10412-10415.

58. Wang, X.; Pan, D.; Weng, D.; Low, C. Y.; Rice, L.; Han, J.; Lu, Y. A General Synthesis of $\mathrm{Cu}-\mathrm{In}-\mathrm{S}$ Based Multicomponent Solid-Solution Nanocrystals with Tunable Band Gap, Size, and Structure. J. Phys. Chem. C 2010, 114, 13406-13413.

59. Kameyama, T.; Douke, Y.; Shibakawa, H.; Kawaraya, M.; Segawa, H.; Kuwabata, S.; Torimoto, T. Widely Controllable Electronic Energy Structure of $\mathrm{ZnSe}-\mathrm{AgInSe} \mathrm{H}_{2}$ Solid Solution Nanocrystals for Quantum-Dot-Sensitized Solar Cells. J. Phys. Chem. C 2014, 118, 29517-29524.

60. Wang, Y.; Xie, Z.; Gotesman, G.; Wang, L.; Bloom, B. P.; Marcus, T. Z.; Oron, D.; Naaman, R.; Waldeck, D. H. Determination of the Electronic Energetics of CdTe Nanoparticle Assemblies on Au Electrodes by Photoemission, Electrochemical, and Photocurrent Studies. J. Phys. Chem. C 2012, 116, 17464-17472.

61. Carlson, B.; Leschkies, K.; Aydil, E.S.; Zhu, X. Y. Valence Band Alignment at Cadmium Selenide Quantum Dot and Zinc Oxide (1010) Interfaces. J. Phys. Chem. C 2008, 112, 8419-8423. 
TOC Graphic

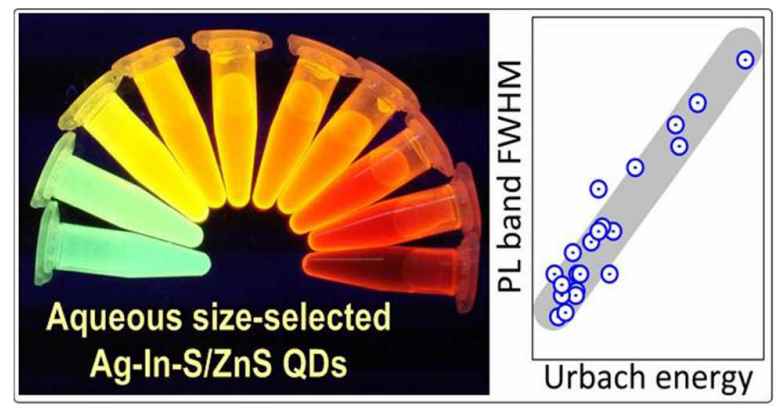

